\title{
Bar resonances and low angular momentum moving groups in the Galaxy revealed by their stellar ages
}

\author{
Chervin F. P. Laporte ${ }^{1,2,3}$, Benoit Famaey ${ }^{4}$ Giacomo Monari $^{4}$, Vanessa Hill ${ }^{2}$, \\ Christopher Wegg ${ }^{2}$, and Ortwin Gerhard ${ }^{5}$ \\ 1 Kavli Institute for the Physics and Mathematics of the Universe (WPI), The University of Tokyo Institutes for Advanced Study \\ (UTIAS), The University of Tokyo, Chiba 277-8583, Japan \\ e-mail: chervin. laporte@ipmu.jp \\ 2 Laboratoire Lagrange, Université Côte d'Azur, Observatoire de la Côte d'Azur, CNRS, Bd de l'Observatoire, 06304 Nice, France \\ 3 Department of Physics \& Astronomy, University of Victoria, 3800 Finnerty Road, Victoria, BC V8P 5C2, Canada \\ ${ }^{4}$ Université de Strasbourg, CNRS UMR 7550, Observatoire Astronomique de Strasbourg, 11 Rue de l'Université, 67000 Strasbourg, \\ France \\ 5 Max-Planck-Institut für Extraterrestrische Physik, Gießenbachstraße 1, 85748 Garching bei München, Germany
}

Received 24 June 2020 / Accepted 27 August 2020

\begin{abstract}
We use the second Gaia data release to dissect the Milky Way disc in phase-space and relative ages. We confirm and report the existence of multiple velocity moving groups at low azimuthal velocities and angular momenta, below Arcturus, regularly separated by $\sim 18-20 \mathrm{~km} \mathrm{~s}^{-1}$ in azimuthal velocity. Such features were predicted to exist more than ten years ago, based on the reaction of the Milky Way to a perturbation in the disc undergoing phase-mixing. These structures appear slightly younger than their phase-space surroundings and reach up to high (solar) metallicities, which argues against an extra-galactic origin. We also identify, in terms of relative age, many of the classical ridges in the plane of azimuthal velocity versus Galactocentric radius, which are traditionally associated with resonance features. These ridges are also younger than their phase-space surroundings in accordance with predictions from recent state-of-the-art cosmological hydrodynamical simulations of Milky Way-like galaxies. We study the response of dynamically young and old stellar disc populations to resonances from an analytic model of a large bar which, remarkably, reproduces qualitatively the trends seen in the data for the classical ridges close to circularity. Our results reinforce the idea that the Galactic disc is being shaped by both internal and external perturbations, along with the fact that while absolute isochrone ages have to be taken with great care, exploring the dynamical structure of the disc in stellar ages, especially with future asteroseismic data, will provide much stronger constraints than metallicity and abundance trends alone.
\end{abstract}

Key words. Galaxy: disk - Galaxy: kinematics and dynamics - solar neighborhood - Galaxy: evolution - Galaxy: structure

\section{Introduction}

The velocity distribution in the solar neighbourhood is far from smooth and it has long been noted to be characterised by substructures. Indeed, a number of moving groups have been known for decades (e.g., Eggen 1965; Dehnen 1998; Famaey et al. 2005), although their various origins are still under debate. Proposed mechanisms range from internal resonances attributed to the bar or spiral arms (e.g. Dehnen 1999; Pérez-Villegas et al. 2017; Monari et al. 2019; Hunt et al. 2019; Fragkoudi et al. 2019), phase-mixing signatures from external perturbations (Minchev et al. 2009; Hunt et al. 2018), and even to extra-galactic accretion origins (Navarro et al. 2004).

This observational fact recently became even more striking thanks to the Gaia DR2 catalogue (Gaia Collaboration 2018a), revealing, in unprecedented detail, prominent arches in the local velocity (Gaia Collaboration 2018b) and action space (Trick et al. 2019a). It is only recently that action-space modeling, despite their promise, have been used to treat perturbations (e.g., Monari et al. 2017a, 2019; Binney 2018), allowing for the recovery of many of the known moving groups at azimuthal velocities, $v_{\phi}$, close to the Sun's velocity. However, in part due to debates and uncertainties about the actual pattern speed of the bar (Monari et al. 2017b, 2019; Pérez-Villegas et al. 2017; Fragkoudi et al. 2019; Trick et al. 2019b), such modeling has not predicted or revealed new substructures in the solar neighbourhood. Instead, using unsharp masking on the Gaia DR2 data, Laporte et al. (2019, their Fig. 13) unveiled the existence of low angular momentum $\left(L_{z}\right)$ velocity substructures, long ago predicted to exist at azimuthal velocities, $v_{\phi}$, below Arcturus from phase-mixing resulting from an external perturbation (Minchev et al. 2009) about 2 Gyr ago. While the regular separation in velocity space in the ridges is a clearly distinct hint of such phase-mixing in the disc, kinematics alone unfortunately does not allow one to posit an in situ or ex situ origin, as has been debated for Arcturus. In order to dispell any further doubt, these structures need to be studied in a complementary space to that of phase-space. While chemistry, such as metallicity or alphaabundances, has been used to study the substructured content of the solar vicinity (e.g. Khanna et al. 2019), the presence of spatial metallicity gradients make interpretative tasks rather difficult. Another issue, especially in the case of Arcturus, is the low 
number of spectroscopically confirmed candidates with the specific kinematics used in the past, which may give the illusion of a tight chemical sequence that suggests the putative existence of an accreted dwarf galaxy (see e.g. Navarro et al. 2004). An addedvalue physical quantity is thus required, which can be provided by differential stellar ages. The recent catalog by Sanders \& Das (2018) provides a compilation of ages derived for about 3 million stars from a combination of legacy spectroscopic surveys with Gaia proper motions, ensuring a full 6D characterisation. While such isochrone ages have to be treated with great care in absolute terms, they can nevertheless be very useful to identify patterns in term of relative ages. Moreover, differential ages as a function of velocity at a given position cannot be affected by selection effects.

Simulations of disc galaxies stratified as a superposition of different stellar age populations, and as a result of different kinematics through the age-velocity dispersion relation (AVR, see, e.g. Fig. 9 of Miglio et al. 2020, for observations), show that younger, colder disc populations react more strongly than older hotter components, whether subject to internal perturbations (e.g., Fragkoudi et al. 2019) or satellite impacts (e.g., Chequers et al. 2018). Thus, characterising the $v_{\phi}-R$ plane in stellar ages would prove particularly useful in the study of the possible disc or accreted origin of low $L_{z}$ moving groups, from Arcturus to lower azimuthal velocities. Moreover, it would also help isolate the signatures of bar resonances in the disc which would manifest themselves at distinct radii depending on the pattern speed and intrinsic structure of the Galactic bar. Typical modeling efforts to study bar resonances in the solar neighbourhood, starting with Dehnen (1999), have represented the bar as a simple $m=2$ quadrupole with varying fixed pattern speeds. However, taking into account higher-order modes in the Fourier decomposition of the bar, Monari et al. (2019) have shown that the situation is more complex. In this Letter, we study the $v_{\phi}-R$ plane in the space of stellar ages to decipher the origin of low $L_{z}$ moving groups as well as the resonances of the bar in the Galaxy as predicted by an analytical action-angle perturbative model based on the large bar of Portail et al. (2017), already used in Monari et al. (2019). In Sect. 2, we present the data used in this study and the actual $v_{\phi}-R$ plane as revealed through stellar ages. In Sect. 3, we compare these results to an analytical bar model to interpret some age structure seen in the data. In Sect. 4, we (re-)analyse the chemical structure of low $L_{z}$ moving groups. We present our conclusions in Sect. 5.

\section{Data and age dissection of phase-space}

The data we use for our analysis comes from the publicly available catalogue of Sanders \& Das (2018), which combines Gaia DR2 data with various current legacy surveys in order to derive isochrone ages for $\sim 3$ million stars. We refer the reader to the original paper for more details. In Fig. 1, we present a series of $v_{\phi}-R$ planes in overdensity (top and bottom left-hand panels), age, and differential ages (top and bottom right-hand panels, respectively). For the density plots, we complement the Sanders \& Das (2018) catalogue with all the Gaia DR2 stars with measured line-of-sight velocities and $\varpi / \sigma \varpi>5$ (RVS). For the RVS stars, we use the distances derived from Bailer-Jones et al. (2018). Given our focus on structures relatively close to the solar neighbourhood, changing to other distance estimates did not change any of our conclusions. For consistency with Sanders \& Das (2018), we transformed from ICRS to Galactocentric coordinates by adopting the position of the Sun at $R_{0}=8.2 \mathrm{kpc}$, a local circular velocity of $v_{\mathrm{c}}\left(R_{0}\right)=$
$240 \mathrm{~km} \mathrm{~s}^{-1}$ and peculiar Solar motion with respect to the local standard of rest of $\left(U_{\odot}, V_{\odot}, W_{\odot}\right)=(11.10,12.24,7.25) \mathrm{km} \mathrm{s}^{-1}$ (Schönrich et al. 2010) and the height of the Sun about the midplane of $z_{\odot}=15 \mathrm{pc}$. Together, this gives us a master catalogue of $\sim 9$ million stars with full 6D phase-space with which to probe the $v_{\phi}-R$ plane.

The presence of the main dominant classical ridges, revealed in number counts by Gaia DR2 (Gaia Collaboration 2018b; Kawata et al. 2018; Antoja et al. 2018; Ramos et al. 2018), including Hercules and the 'hat', is evident in Fig. 1, together with the additional lower velocity ridges below Arcturus, all separated by $\sim 18-20 \mathrm{~km} \mathrm{~s}^{-1}$ in density $(\delta=\rho / \bar{\rho}-1)$ but also in the age moment map of the $v_{\phi}-R$ plane (denoted by the black arrows in Fig. 1).

Two of them (denoted 'Snoop' and 'Chaka') were predicted by Minchev et al. (2009) to lie between Arcturus and 'Herbie'/K08'. We also note the presence of a few low $L_{z}$ moving groups further below Herbie/K08 (Fig. 1 bottom left) such as the ridges at $v_{\phi} \sim 70,50,30 \mathrm{~km} \mathrm{~s}^{-1}$ (which we label 'Ella', 'Dizzy', 'Bird'), digging deeper in the halo-dominated region of velocity-space. Some of these ridges were tentatively seen in Klement et al. (2008) however the authors did not further comment on their result due to the paucity of tracers used at the time. While the Gaia DR2 sample increased this, given the limited complementary data available (age, metallicity, abundance), we leave them as curiosities (hence the dashed arrows in Fig. 1) to be explored in detail with future Data Releases.

Although low $L_{z}$ moving groups such as Arcturus have been proposed to originate from an extra-galactic origin, we see that the ridges from Arcturus down to Ella all have stellar ages that are systematically younger than the surrounding population. This is clear evidence against an extra-galactic origin for such structures, which otherwise should have been, if anything, older than the surroundings. Combined with the regularity in intervals between ridges in velocity space, this clearly argues for a relic sign of a perturbation to the Milky Way disc which is currently undergoing phase-mixing (Minchev et al. 2009). Proposed scenarios include internal perturbations from transient spiral arms (Hunt et al. 2019), a slowing bar (Chiba et al. 2019), or external perturbations by a satellite which naturally seed spirals and give rise to ridges (Purcell et al. 2011; Gómez et al. 2012, 2013; Laporte et al. 2018, 2019). Disentangling between these processes acting on Galactic discs, which may act simultaneously, likely requires the development of tools beyond those that are typically used (test-particles/ $N$-body simulations) and, thus, this is beyond the scope of this Letter.

Amongst the classical ridges, we notice the 'hat' (the L2 ridge from Ramos et al. 2018) - identified as the outer Lindblad resonance (OLR) in our bar model in the next section - which looks split in density, as was already noted in Laporte et al. (2019; see their Fig. 13, top panel) with possibly an outwardinward radial motion split (Laporte et al. 2019; Fragkoudi et al. 2019). What follows, from large to lower $L_{z}$, is Sirius, Hyades, and Hercules, which we further analyse. In the space of relative ages, Sirius is very prominent through a wide range of radii $(6<R / \mathrm{kpc}<12)$. In contrast, the 'hat' stands out most strongly at radii beyond $R \sim 9 \mathrm{kpc}$ and Hercules appears (albeit more weakly than Sirius) in the solar neighbourhood and picks up more strongly towards $R \sim 7 \mathrm{kpc}$, both in terms of differential ages and in the global density in the $v_{\phi}-R$ plane. These interesting trends are best appreciated in the differential age map of the $v_{\phi}-R$ plane.

\footnotetext{
1 Herbie was in fact uncovered using phase-space information alone using the first RAVE catalogue by Klement et al. (2008).
} 

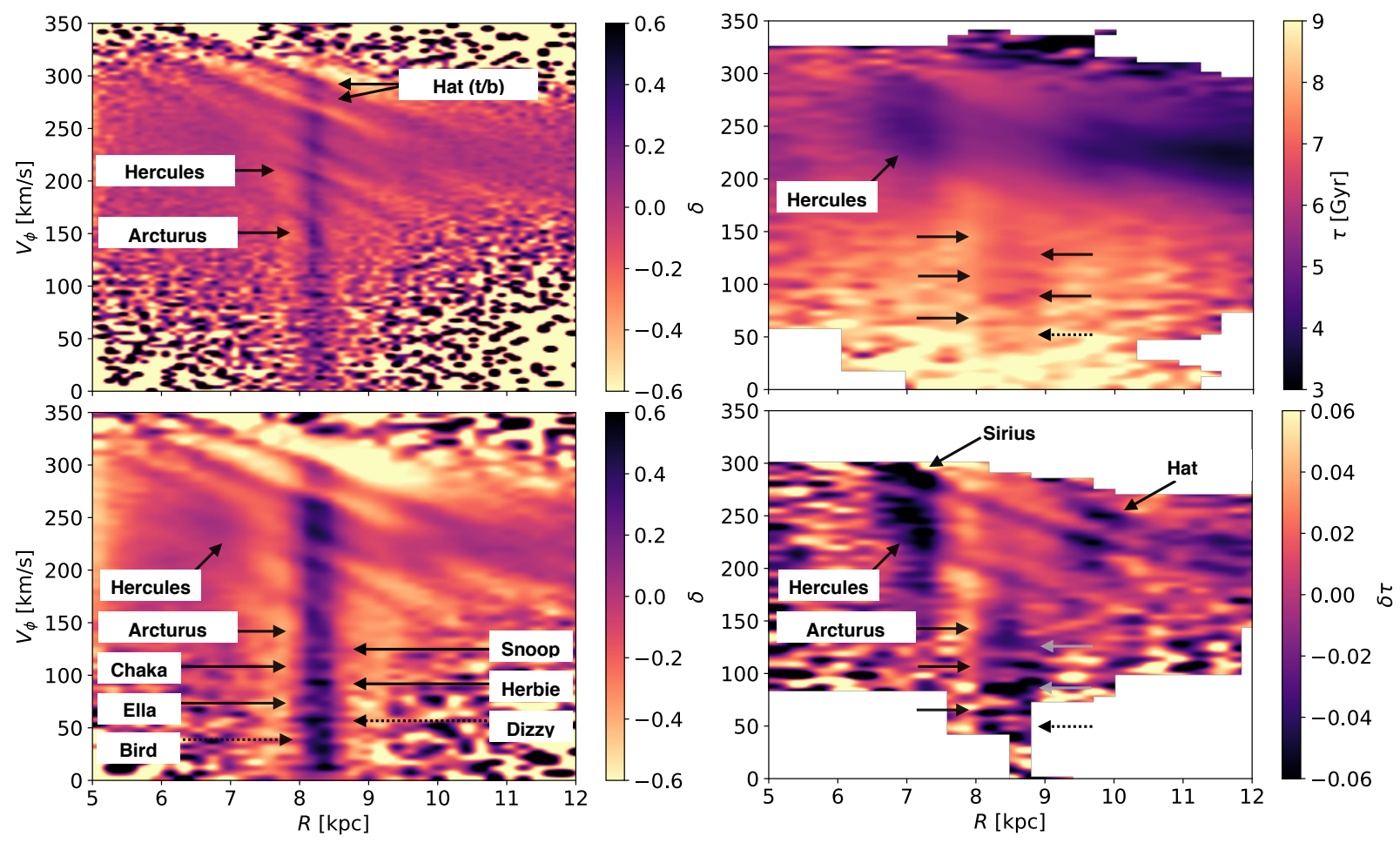

Fig. 1. Top left: unsharp masked $\delta\left(v_{\phi}-R\right)$ plane for the Gaia DR2 and Sanders \& Das (2018) master catalog ( 9 million sources with 6D phasespace information). The bin size is $\left(\Delta_{\mathrm{R}}, \Delta v_{\phi}\right)=\left(0.1 \mathrm{kpc}, 3 \mathrm{~km} \mathrm{~s}^{-1}\right)$ and helps visualise the finer parts of the classical ridges (Sirius, Hyades, Hercules). We note that the 'hat' seems to be split in two (which is expected at the OLR from the orbits trapped at the resonance and the linear deformation of velocity space below the resonance). Arcturus also stands out. Bottom left: similar to the top left figure but with a bin size, $\left(\Delta_{\mathrm{R}}, \Delta v_{\phi}\right)=\left(0.2 \mathrm{kpc}, 5 \mathrm{~km} \mathrm{~s}^{-1}\right)$, that is meant to help low $L_{z}$ moving groups below Arcturus stand out. These are separated by $\sim 18-20 \mathrm{~km} \mathrm{~s}{ }^{-1}$ consistent with the Minchev et al. (2009)'s predictions (Snoop, Chaka, Herbie/K08 with 2170, 890 and 530 stars within 4 bins), the series extends further to lower velocities (e.g. 'Ella' $v \sim 70 \mathrm{~km} \mathrm{~s}^{-1}$, 'Dizzy' $v \sim 50 \mathrm{~km} \mathrm{~s}^{-1}$, and even perhaps what we denote 'Bird' at $\sim 30 \mathrm{~km} \mathrm{~s}$ ). Top right: age moment $\tau$ of the $v_{\phi}-R$ plane for the Sanders \& Das (2018) catalog. Ridges from Hercules, Sirius and the 'hat' (OLR) appear strongly. low $L_{z}$ moving groups (Arcturus, Snoop, Chaka, Herbie, Ella) also show up as slightly younger patches. We caution against interpreting those absolute ages as a function of radius, as selection effects might be important. What is more useful is to compare ages as a function of velocities at a fixed radius. Bottom right: differential age $(\delta \tau=\tau / \bar{\tau}-1)$ distribution in the $v_{\phi}-R$ plane. Low $L_{z}$ ridges show up as slightly but prominently younger than their surrounding. The 'hat' (OLR) shows a prominent signal at radii beyond the $\operatorname{Sun}(9<R / \mathrm{kpc}<11)$. Sirius is present across the whole range $(7<R / \mathrm{kpc}<11)$, and Hercules is particularly strong at radii smaller than $R_{0}$ with an extension out to $9.5 \mathrm{kpc}$.

\section{Analytic bar model}

In this section, we consider the analytic model developed by Monari et al. (2017a), aimed at studying the response of a disc composed of a dynamically young (low velocity dispersion) and old (high velocity dispersion) stellar populations to the large bar model of Portail et al. (2017) with a pattern speed of $\Omega_{\mathrm{b}}=$ $39 \mathrm{~km} \mathrm{~s}^{-1} \mathrm{kpc}^{-1}$. This dynamical model is based on the number density of red clump stars in the bulge and long bar (cf. Wegg et al. 2015) and the line-of-sight velocities of large samples of bulge giants. Already in Monari et al. (2019), this model was shown to reproduce a number of ridges in the Gaia DR2 data which they associated with the OLR, corotation (CR), as well as the 4:1 and 6:1 resonances from higher order Fourier modes of the bar potential.

We define a quasi-isothermal (Binney 2010) unperturbed distribution function in action space to represent a young and cold $\left(\sigma_{\mathrm{R}}=30 \mathrm{~km} \mathrm{~s}^{-1}\right)$, as well as a hotter and older $\left(\sigma_{\mathrm{R}}=50 \mathrm{~km} \mathrm{~s}^{-1}\right)$ disc stellar population. Then we use a first canonical transformation to move to the slow and fast action-angle variables, which allows us to determine the regions of phase-space where orbits are trapped at resonances. In the vicinity of these resonant regions, and after averaging over the fast angles, the dynamics of the slow variables is that of a pendulum, for which we make a new canonical transformation to pendulum action-angle vari- ables. We then phase-mix the original unperturbed distribution function over the pendulum angles to obtain the perturbed distribution function in the different resonant zones. We can then compute the perturbed distribution function at different positions and velocities, making use of the epicyclic approximation. We refer the reader to Monari et al. $(2017 \mathrm{a}, 2019)$ for more details of the method. We note that it gives a very good representation of orbits trapped at resonances, but is not the best possible description of the linear deformation zones. In Fig. 2, we present the variations in density of the $v_{\phi}-R$ plane resulting from the response of both the cold (young) and hot (old) stellar populations to the bar potential of Portail et al. (2017). As expected, the features are marked much more clearly for the dynamically young population. Interestingly, this is particularly true for the $4: 1$ resonance, which corresponds to the Sirius ridge, which is also seen very prominently in the data as a young feature in a large range of radii. Moreover, the density contrast of the ridge associated with corotation is most prominent for young stars around $R \sim 7 \mathrm{kpc}$, which is also where the Hercules ridge appears most clearly both in global density and as a young feature in the data. The 'hat' feature, which corresponds to the bar's OLR in this model, appears prominently for the young and cold population at $R \sim 9 \mathrm{kpc}$, where it also peaks in differential age in the data. This analytical model thus provides a remarkable qualitative match to the trend uncovered in the data. 

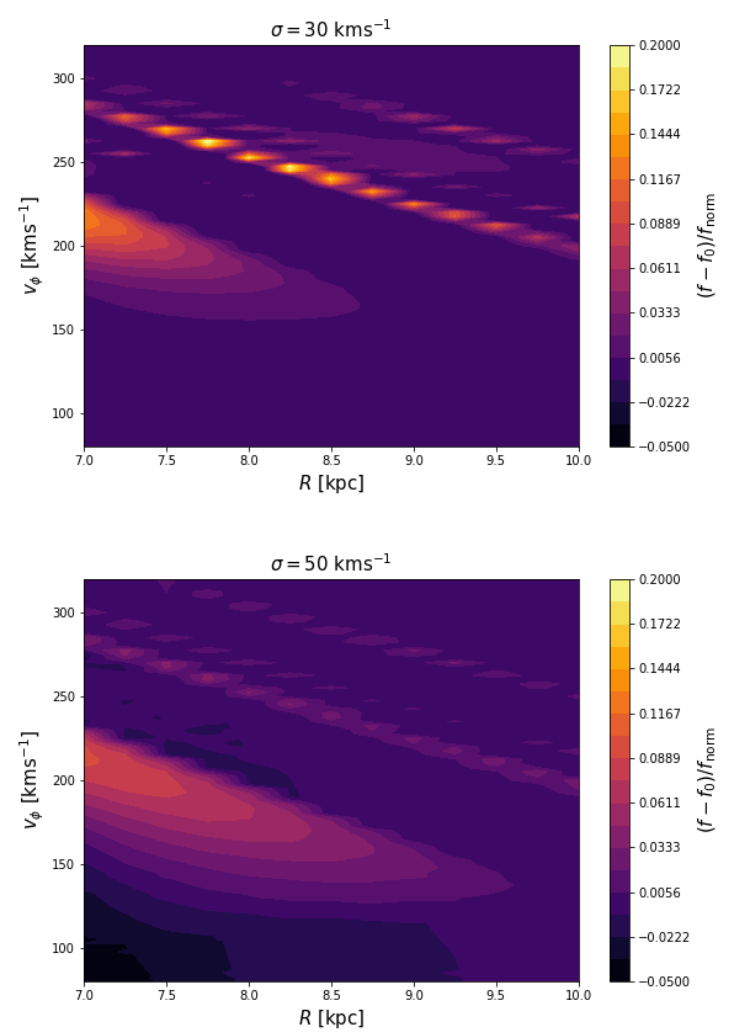

Fig. 2. Normalised perturbed distribution function in the presence of the large bar perturbation of Portail et al. (2017). Top panel: for an unperturbed distribution function representing a young and cold disc stellar population with $\sigma_{\mathrm{R}}=30 \mathrm{~km} \mathrm{~s}^{-1}$ at the Galactocentric solar radius $R_{0}$. Bottom panel: for an older and hotter disc population with $\sigma_{\mathrm{R}}=50 \mathrm{~km} \mathrm{~s}^{-1}$ at the Galactocentric solar radius $R_{0}$.

\section{Arcturus and moving groups of the thick disc}

We note in Sect. 2 that the ridges from Arcturus down to Ella all have stellar ages that appear slightly but systematically younger than the surrounding population, which, combined with the regularity in intervals between ridges in velocity space, argues for a relic sign of a perturbation to the Milky Way disc. In this section, we use chemistry to confirm this picture in the case of Arcturus and low $L_{z}$ moving groups.

Using the catalogue of metal-poor stars from Gratton et al. (2003, hereafter GCCLB), Navarro et al. (2004, hereafter N04) selected stars in the solar neighbourhood from the Arcturus group following two criteria: $1100<L_{z} / \mathrm{km} \mathrm{s}^{-1} \mathrm{kpc}<1300$ and $\left|v_{z}\right|<50 \mathrm{~km} \mathrm{~s}^{-1}$. They found a tight sequence in the $([\alpha / \mathrm{Fe}]$, $[\mathrm{Fe} / \mathrm{H}])$ plane with respect to the full GCCLB sample, which they used as an argument for a possible extra-galactic origin. However, it must be noted that the sample used was modest in size and biased towards low metallicities, which stop around $[\mathrm{Fe} / \mathrm{H}] \sim-0.6$. As we shall see, such biases easily mimic a tight sequence. Using the APOGEE DR14 catalogue (Abolfathi et al. 2018), we carry out the same analysis as in N04. In Fig. 3, we present the $([\mathrm{Mg} / \mathrm{Fe}],[\mathrm{Fe} / \mathrm{H}])$ plane for Arcturus and the low $L_{z}$ moving groups. These were selected as follows in the APOGEE catalogue:

$-d<0.4 \mathrm{kpc}$

$-|z|<1 \mathrm{kpc}$

$-1100<L_{z} / \mathrm{km} \mathrm{s}^{-1} \mathrm{kpc}<1300$ and $\left|v_{z}\right|<50 \mathrm{~km} \mathrm{~s}^{-1}$, for Arcturus

- $952<L_{z} / \mathrm{km} \mathrm{s}^{-1} \mathrm{kpc}<929$, for Chaka

$-624<L_{z} / \mathrm{km} \mathrm{s}^{-1} \mathrm{kpc}<860$, for Herbie.

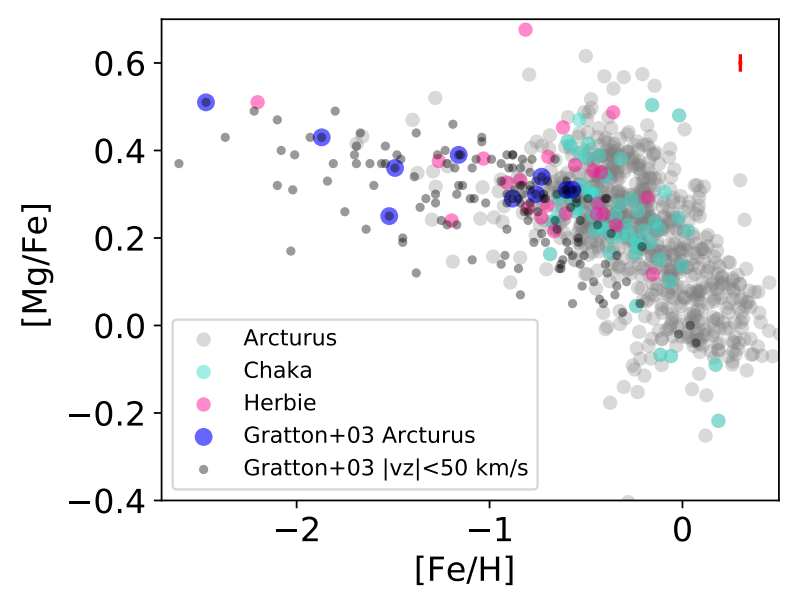

Fig. 3. $[\mathrm{Mg} / \mathrm{Fe}]$ vs $[\mathrm{Fe} / \mathrm{H}]$ plot for stars in Arcturus (as selected by N04 which intrinsically includes Snoop), Chaka and Herbie from APOGEE in thick grey, turquoise, and magenta dots. We also overlay the original stars used in N04 from the GCCLB dataset. We note that this dataset of metal poor stars was biased and falls at the boundary with the disc as well as the continuation of the chemically thick disc. The larger APOGEE DR14 sample extends just as much into the metal poor end probed by GCCLB and together with the N04 Arcturus sample gives a more complete census on the origin of Arcturus from the chemical side alone. Typical errorbar is marked in red.

We note that APOGEE also finds stars in the same region probed by N04, however the Arcturus magnesium sequence is no longer tight but rather (1) broad; (2) with no signs of a knee; and (3) extends well into the thick disc with a contribution from the chemical thin disc. The other low $L_{z}$ moving groups ${ }^{2}$ (e.g. Chaka, Herbie), on the other hand, are predominantly composed of thick disc stars and occupy the same space as Arcturus.

While chemistry has also been used as an argument in favour of an internal origin for the structure, these came from separate analyses (e.g Kushniruk \& Bensby 2019) which did not compare to the original N04 results to understand the differing conclusions. While chemistry is a useful quantity to study, it should nonetheless be supplemented by stellar ages as a third complementary and final check. Here, we have shown that the ages of those low $L_{z}$ moving groups tend to be slightly lower relative to their surroundings. Indeed, even the thick disk displays an AVR (Miglio et al. 2020). While the association between phase-space structures and younger structures in terms of relative age is a striking result, we caution against the use of isochrone ages to evaluate the 'absolute' age of stars in these structures. The latter will need future asteroseismic data for large samples in order to quantify the exact age of these structures (Miglio et al. 2020).

\section{Discussion and conclusions}

Identifying signatures associated with the bar in the solar neighbourhood in phase-space alone is notoriously difficult (see Trick et al. 2019b). Other diagnostics such as metallicity, alphaabundances, and ages have been proposed as a way of identifying prominent ridges associated with the OLR, for example (e.g. Fragkoudi et al. 2020). These have been investigated in GALAH for metallicity and abundances (Khanna et al. 2019). However, stellar ages remain the most physically interesting quantity (albeit less precisely measured) both in terms of probing the growth history of the disc and its correlation with the dynamics through the age-velocity dispersion relation (AVR;

2 The broad angular momentum selection in N04 for Arcturus also comprises Snoop, not shown here for clarity in Fig. 3. 
e.g. Sanders \& Das 2018; Miglio et al. 2020). Here, we explore, for the first time, the $v_{\phi}-R$ plane in the space of ages. Interestingly, our findings highlight and confirm predictions from state-of-the-art simulations of Milky Way-like systems that regions associated with resonances of the bar should, on average, be younger than the surrounding phase-space (Fragkoudi et al. 2020). We compare the data to predictions from an analytic model based on the large bar of Portail et al. (2017), as in Monari et al. (2019). The model is able to qualitatively predict the spatial variations and locations of differential age peaks of known classical ridges associated with bar resonances such as the 'hat' (OLR), Sirius (4:1 resonance) and Hercules (CR). Although this Letter is a first exploration of the signature of the bar in different stellar age populations, we note a few caveats. We assumed that the bar has a constant pattern speed with the parameters derived by Portail et al. (2017). We left out the contribution of spiral arms and the possibility that the bar is currently reconnecting with a spiral arm and displays fast pattern speed variations (Petersen et al. 2019; Hilmi et al. 2020). It will be interesting to investigate how such models with varying pattern speed compare to the data presented here.

Another novel result from analysing the $v_{\phi}-R$ plane in differential ages is the complementary confirmation of low $L_{z}$ (i.e. high-velocity with respect to the Sun) moving groups predicted by a model of Minchev et al. (2009) below Arcturus: Snoop, Chaka, Herbie/K08, and Ella - the latter two having been previously noted in Klement et al. (2008), but at the time, not as a significant detection in the case of Ella (only two stars). The association we found between phase-space structures and slightly younger structures in terms of relative age is striking and it argues for a relic sign of a perturbation to the Milky Way disc. When combined with the wide $[\mathrm{Mg} / \mathrm{Fe}]$ and $[\mathrm{Fe} / \mathrm{H}]$ distributions for Arcturus, Chaka, Herbie, and Ella, which go up to solar, it is clear there is no room to argue for an extra-galactic origin for these components. For reference, satellites with mean metallicities in the range of $-0.9<[\mathrm{Fe} / \mathrm{H}]<-0.5$ would imply progenitors with stellar masses between $9 \times 10^{8}<M_{*} / M_{\odot}<4 \times 10^{9}$ (Kirby et al. 2013) and would be in stark conflict with the stellar halo mass budget $M_{\mathrm{sh}} \sim 10^{9} M_{\odot}$ (Deason et al. 2019).

Although Arcturus has been argued in the past to be a substructure associated with an extra-galactic orign (N04), this interpretation was challenged in Minchev et al. (2009), who proposed an alternative interpretation as the response of the disc to a perturber. This was given further credence with Gaia DR2 in Laporte et al. (2019), and also corroborated by Kushniruk \& Bensby (2019). Here, we provide the final argument in favour of an internal origin through a re-analysis of the samples used in N04 complemented with APOGEE data (Abolfathi et al. 2018). We show, in particular, that the sequence argued in N04 does not show a sharp knee but, rather, it is broader than that highlighted using only GCCLB and continues well into the thick disc regimes at $[\mathrm{Fe} / \mathrm{H}] \sim-0.6$ for similarly kinematically selected stars as in N04 (complementing the GCCLB sample). This conclusion holds as well for the other lower $L_{z}$ moving groups. As an outlook into the future, Gaia DR3 will provide radial velocities and stellar parameters for about 40 million stars and this number is expected to increase with surveys such as WEAVE, SDSS-V and 4MOST, allowing for a better decomposition of the phase-space structure of the disc (as well as in metallicity and $\alpha$-elemental abundances). Together with stellar ages, this should enable us to better understand the interplay between external and internal perturbations in shaping the disc of the Galaxy and to constrain the structure of the central region of the Milky Way. It is already clear that asteroseismology will play a major role in this endeavour (Miglio et al. 2017, 2020; Huber et al. 2019).
Acknowledgements. CL thanks the Observatoire de la Côte d'Azur for hosting him and J. F. Navarro for discussions. This work was supported in part by World Premier International Research Center Initiative (WPI Initiative), MEXT, Japan. BF and GM acknowledge funding from the Agence Nationale de la Recherche (ANR project ANR-18-CE31-0006 and ANR-19CE31-0017) and from the European Research Council (ERC, grant agreement No. 834148). This project has received funding from the European Research Council (ERC) under the European Union's Horizon 2020 research and innovation programme (grant agreement No. 852839). This work has made use of data from the European Space Agency (ESA) mission Gaia (https://www . cosmos.esa.int/gaia), processed by the Gaia Data Processing and Analysis Consortium (DPAC, https://www. cosmos.esa.int/web/gaia/dpac/ consortium). The new ridges were named in honour of Snoop Dogg, Chaka Khan, Herbie Hancock, Ella Fitzgerald, Dizzy Gillespie and Charlie "Bird" Parker.

\section{References}

Abolfathi, B., Aguado, D. S., Aguilar, G., et al. 2018, ApJS, 235, 42 Antoja, T., Helmi, A., Romero-Gómez, M., et al. 2018, Nature, 561, 360 Bailer-Jones, C. A. L., Rybizki, J., Fouesneau, M., Mantelet, G., \& Andrae, R. 2018, AJ, 156, 58

Binney, J. 2010, MNRAS, 401, 2318

Binney, J. 2018, MNRAS, 474, 2706

Chequers, M. H., Widrow, L. M., \& Darling, K. 2018, MNRAS, 480, 4244

Chiba, R., Friske, J. K. S., \& Schönrich, R. 2019, MNRAS, submitted [arXiv:1912.04304]

Deason, A. J., Belokurov, V., \& Sanders, J. L. 2019, MNRAS, 490, 3426

Dehnen, W. 1998, AJ, 115, 2384

Dehnen, W. 1999, ApJ, 524, L35

Eggen, O. J. 1965, Moving Groups of Stars (Chicago: University of Chicago Press), 111

Famaey, B., Jorissen, A., Luri, X., et al. 2005, A\&A, 430, 165

Fragkoudi, F., Katz, D., Trick, W., et al. 2019, MNRAS, 488, 3324

Fragkoudi, F., Grand, R. J. J., Pakmor, R., et al. 2020, MNRAS, 494, 5936

Gaia Collaboration (Brown, A. G. A., et al.) 2018a, A\&A, 616, A1

Gaia Collaboration (Katz, D., et al.) 2018b, A\&A, 616, A11

Gómez, F. A., Minchev, I., O'Shea, B. W., et al. 2012, MNRAS, 423, 3727

Gómez, F. A., Minchev, I., O'Shea, B. W., et al. 2013, MNRAS, 429, 159

Gratton, R. G., Carretta, E., Claudi, R., Lucatello, S., \& Barbieri, M. 2003, A\&A, 404, 187

Hilmi, T., Minchev, I., Buck, T., et al. 2020, MNRAS, 497, 933

Huber, D., Basu, S., Beck, P., et al. 2019, ArXiv e-prints [arXiv:1903.08188]

Hunt, J. A. S., Hong, J., Bovy, J., Kawata, D., \& Grand, R. J. J. 2018, MNRAS, 481,3794

Hunt, J. A. S., Bub, M. W., Bovy, J., et al. 2019, MNRAS, 490, 1026

Kawata, D., Baba, J., Ciucă, I., et al. 2018, MNRAS, 479, L108

Khanna, S., Sharma, S., Tepper-Garcia, T., et al. 2019, MNRAS, 489, 4962

Kirby, E. N., Cohen, J. G., Guhathakurta, P., et al. 2013, ApJ, 779, 102

Klement, R., Fuchs, B., \& Rix, H. W. 2008, ApJ, 685, 261

Kushniruk, I., \& Bensby, T. 2019, A\&A, 631, A47

Laporte, C. F. P., Johnston, K. V., Gómez, F. A., Garavito-Camargo, N., \& Besla, G. 2018, MNRAS, 481, 286

Laporte, C. F. P., Minchev, I., Johnston, K. V., \& Gómez, F. A. 2019, MNRAS, 485,3134

Miglio, A., Chiappini, C., Mosser, B., et al. 2017, Astron. Nachr., 338, 644

Miglio, A., Chiappini, C., Mackereth, T., et al. 2020, A\&A, submitted [arXiv:2004.14806]

Minchev, I., Quillen, A. C., Williams, M., et al. 2009, MNRAS, 396, L56

Monari, G., Famaey, B., Fouvry, J.-B., \& Binney, J. 2017a, MNRAS, 471, 4314

Monari, G., Kawata, D., Hunt, J. A. S., \& Famaey, B. 2017b, MNRAS, 466, L113

Monari, G., Famaey, B., Siebert, A., Wegg, C., \& Gerhard, O. 2019, A\&A, 626, A41

Navarro, J. F., Helmi, A., \& Freeman, K. C. 2004, ApJ, 601, L43

Pérez-Villegas, A., Portail, M., Wegg, C., \& Gerhard, O. 2017, ApJ, 840, L2

Petersen, M. S., Weinberg, M. D., \& Katz, N. 2019, MNRAS, submitted [arXiv:1903.08203]

Portail, M., Gerhard, O., Wegg, C., \& Ness, M. 2017, MNRAS, 465, 1621

Purcell, C. W., Bullock, J. S., Tollerud, E. J., Rocha, M., \& Chakrabarti, S. 2011,

Nature, 477, 301

Ramos, P., Antoja, T., \& Figueras, F. 2018, A\&A, 619, A72

Sanders, J. L., \& Das, P. 2018, MNRAS, 481, 4093

Schönrich, R., Binney, J., \& Dehnen, W. 2010, MNRAS, 403, 1829

Trick, W. H., Coronado, J., \& Rix, H.-W. 2019a, MNRAS, 484, 3291

Trick, W. H., Fragkoudi, F., Hunt, J. A. S., Mackereth, J. T., \& White, S. D. M. 2019b, MNRAS, submitted [arXiv:1906.04786]

Wegg, C., Gerhard, O., \& Portail, M. 2015, MNRAS, 450, 4050 